\title{
Science, Technologie et Développement Economique : Des enjeux impérieux et contrariés
}

\author{
Science, Technology and Economic Development: Important and critical
} issues

\author{
Vanessa Casadella ${ }^{1}$ \\ ${ }^{1}$ Université Picardie Jules Verne, Laboratoire CRIISEA, RRI (Réseau de Recherche sur l'Innovation) \\ vanessa.casadella@u-picardie.fr
}

\begin{abstract}
RÉSUMÉ. La Science et technologie (S\&T) sont des enjeux majeurs pour le développement économique et le progrès social. Inscrits dans les ODD de 2015 pour l'Agenda de 2030, leur légitimité n'est pas récente dans la problématique des pays en développement ou en transition. Néanmoins, si leur bienfondé était lié à l'appropriation et la diffusion d'une technologie stricto sensu, le regard actuellement posé sur elles est plus large et lié à la construction de compétences et de capacités. Le présent article propose de revisiter l'état de l'art de la science et la technologie comme interdépendantes du développement économique, avant d'exposer le lien, important mais contrarié dans les théories actuelles du développement économique.

ABSTRACT. Science and technology (S\&T) are major challenges for economic development and social progress. Inscribed in the SDGs of 2015 for the 2030 Agenda, their legitimacy is not recent in the issue of developing countries or countries in transition. However, if their merits were linked to the appropriation and dissemination of technology stricto sensu, the current view of them is broader and linked to the construction of competencies and capacities. This article proposes to revisit the state of the art of science and technology as interrelated with economic development, before exposing the important but thwarted link in current theories of economic development.

MOTS-CLÉS. Science, Technologie, Développement Economique, Capacités, Politique d'Innovation, Pays en Développement.

KEYWORDS. Science, Technology, Economic Development, Capacity, Innovation Policy, Developing Countries.
\end{abstract}

Une note de 2017 de la CNUCED [ONU, 17] rappelait à juste titre que les capacités des pays en développement, la science, la technologie et l'innovation peuvent jouer un rôle moteur dans l'amélioration de la productivité et la croissance économique, promouvoir l'inclusion sociale et favoriser la durabilité environnementale. En effet, investir dans la science et la technologie est devenu essentiel pour le développement économique et le progrès social. Aujourd'hui, la Recherche et Développement $(\mathrm{R} \& \mathrm{D})$ contribue au progrès économique et social tout en préservant l'environnement et édifiant des sociétés intrinsèquement plus inclusives. Les objectifs de développement durable (ODD), énoncés en 2015 par l'ONU dans le cadre de l'Agenda 2030, sont l'un des projets les plus transformateurs que la communauté mondiale ait lancé depuis des décennies. La science et la technologie y jouent un rôle moteur dans le changement économique et social.

L'ONU a défini en 201517 objectifs de développement durable donnant la marche à suivre pour assurer un "monde meilleur et plus durable pour tous ». Ces objectifs, interconnectés, doivent être atteints d'ici 2030. Ils touchent à des domaines très divers tels que la pauvreté, la faim, la santé et le bien-être, l'éducation, le genre, l'eau potable, l'énergie, le travail décent, l'industrie et l'innovation, les inégalités, les villes durables, la consommation, le changement climatique, la vie aquatique et terrestre, la paix et la justice, ainsi que les partenariats pour la réalisation de ces objectifs. L'objectif 9 , lié à la thématique du progrès technique, est tourné vers l'innovation et la $R \& D$, au profit de solutions durables. Selon le PNUD, ces ODD représentent une forme d' " appel mondial » à agir pour éradiquer la pauvreté, protéger la Planète et faire en sorte que la population mondiale puisse vivre dans la paix et la prospérité. Si ces ODD représentent un véritable plan d'action sur 169 cibles, 
nécessitant une approche multi-acteurs entre 193 pays identifiés, leur ambition ne semble pas à la portée de tous les pays, dont ceux les plus touchés par la famine, les guerres inter-ethniques, les conflits politiques ou religieux. Un exemple représentatif est celui de l'accès à la justice, largement inaccessible à beaucoup de pays africains [LOG, 17]. La réalité dépasse ici très nettement l'ambition souhaitée. Par ailleurs, s'est posée la question de l'évaluation des capacités statistiques relatives aux ODD afin d'uniformiser les systèmes statistiques nationaux et les rendre cohérents les uns aux autres. Cette question est en cours de traitement par des organisations internationales, telles que la FAO (Food and Agriculture Organization). Cette dernière, rattachée à l'ONU, pointait récemment du doigt le bilan négatif lié à la faim, sécurité alimentaire et nutrition : 820 millions de personnes souffrent encore de la faim en 2019. A ce titre, et dans le cadre du suivi des progrès accomplis liés à l'alimentation et à l'agriculture durable, les progrès demeurent largement insuffisants et le monde est loin d'atteindre les cibles proposées d'ici 2030 [FAO, 20]. Il reste clair que ces 17 objectifs ne pourront pas tous être réalisables d'ici 2030, déjà car (très) nombreux, mais surtout au regard de la grande diversité des paysages économiques globaux, certains pays ayant une plus grande source de progression que d'autres, et sur certains domaines donnés.

\section{Encadré 1. Les Objectifs du Développement Durable (ODD) de l'ONU (2015)}

Au fil des décennies, les systèmes technologiques et d'innovation se sont diversifiés grâce à l'intérêt porté par des chercheurs en économie et en management de l'innovation, mais surtout grâce au développement de réseaux internationaux de recherche tels que Globelics ou le Réseau de Recherche sur l'Innovation (réseaux internationaux), Lalics (réseau sud-américain) et Africalics (réseau africain). Les systèmes technologiques et d'innovation ne représentent plus uniquement des systèmes de R\&D, mais des systèmes portés par une forte dimension sociétale, environnementale, économique et sociale. Les innovations frugales, les technologies bottom up sont particulièrement appréciées dans un contexte où les nouvelles technologies réduisent les prix des biens et services auxquels elles sont appliquées. Les téléphones portables sont un exemple clair de l'impact profond que peuvent avoir certaines nouvelles technologies. Ils ont fourni aux personnes pauvres dans les pays en développement l'accès aux communications à longue distance, sans devoir passer par des investissements coûteux dans des lignes fixes et d'autres infrastructures. De même, les applications bancaires mobiles fournis par les téléphones portables ont rendu possible l'accès aux services financiers dans des régions éloignées sans aucune succursale bancaire. Néanmoins, si leur pertinence semble à ce jour légitimée, les liens entre la science et la technologie $(\mathrm{S} \& \mathrm{~T})$ et le développement économique n'ont pas été toujours évidents à reconnaitre. Tout d'abord car l'objectif initial principal de la S\&T était, par le biais des thèses sur le transfert technologique, d'absorber des connaissances technologiques stricto sensu, mais surtout car les modèles inclusifs portés autour de la S\&T ne se sont développés que tardivement, autour de la notion de construction de capacités et compétences. Aujourd'hui, c'est bien un angle d'approche très large qui est recherché à travers la S\&T, pour parvenir à un meilleur développement économique.

Dans cette perspective, notre première partie reviendra sur la chronologie des travaux portés sur la dimension de la science et technologie comme enjeux du progrès économique (1). Notre démarche méthodologique s'appuiera ici sur un constant empirique relatif aux pays en développement et une revue de littérature sur le sujet. Notre seconde partie analysera la portée théorique de ces liens : science/ technologie et développement économique, peu entretenus dans la littérature en économie de l'innovation, mais particulièrement importants à faire valoir dans notre actualité, politique et internationale, marquée par une dimension inclusive très forte (2). 


\section{La Science et Technologie comme enjeux du Développement Economique : Capacité versus Transfert}

\subsection{La faiblesse de la science et technologie dans les Pays en Développement}

Si les dénommés «pays en développement » sont marqués par une forte hétérogénéité en termes de processus de croissance et de développement économique, il n'en demeure pas moins que les données disponibles sur la science et technologie reflètent majoritairement une faiblesse de leurs institutions de S\&T et dépenses en R\&D. Les causes sont multiples : fragiles politiques volontaristes sur la S\&T liées à une non-priorisation de ce secteur, conflits religieux ou politiques, fuite des cerveaux, absence d'évaluation complète des besoins dans les secteurs public et privé, etc.

Les mesures relatives à la science et technologie sont, elles-aussi, très hétérogènes. Si les institutions internationales s'accordent majoritairement à faire valoir la $R \& D$ ou les brevets comme principaux outputs de la S\&T, les travaux en économie de l'innovation [VIO, 02] englobent des indicateurs plus larges liés au système éducatif, à l'acquisition technologique, aux efforts en termes de $R \& D$ et aux dépôts de brevets. A en regarder uniquement les dépenses en R\&D (Figure 1), l'Afrique Subsaharienne et l'Asie centrale sont les régions disposant d'une maigre contribution en termes dépenses de R\&D. Plus précisément, les moyennes régionales pour la part du PIB consacré aux activités de R\&D représentent $0,2 \%$ pour l'Asie centrale et $0,4 \%$ pour l'Afrique sub-saharienne [UNE, 19].Même si de forts contrastes sont à noter sur la répartition de l'Afrique Sub-saharienne en termes de dépenses en R\&D, l'Afrique rencontre de larges difficultés pour rester en course dans un environnement mondial de plus en plus concurrentiel. De plus, les I.D.E (investissements directs à l'étranger) demeurent insuffisamment contributeurs à l'économie réelle et aux équilibres externes. Quant à l'Asie centrale (exception faite du Kazakhstan), son volume d'I.D.E dans le secteur de la R\&D est, lui aussi, trop faible et la mise en place de gouvernements autoritaires ne va pas en faveur de son attrait potentiel.

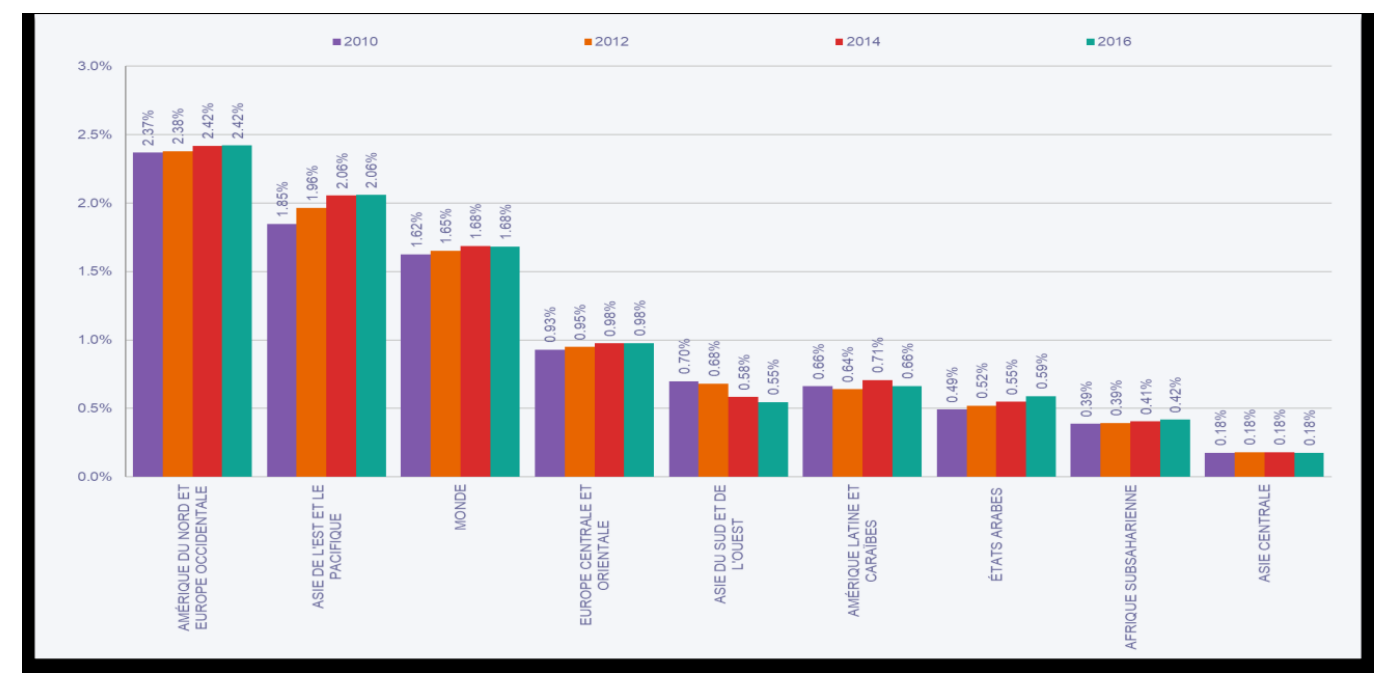

Source : UNESCO, 2019

Figure 1. Les régions les plus intensives en $R \& D$ (DIRD en \% par région)

Chaque pays définit en matière de S\&T un ensemble de priorités sur la mise en place de politiques horizontales (subventions/règlementations), verticales (renforcement des industries existantes) ou sectorielles (technologies endogènes). Dans certains cas, les priorités reflètent le contexte national mais dans grand nombre de cas, l'approche est standardisée et s'inspire de domaines où le pays a la capacité de se positionner à la frontière de la connaissance. Dans tous les cas, les politiques de S\&T sont variables d'un pays à l'autre et l'aperçu de l'intensité mondiale en R\&D le prouve aisément (Figure 2). Les continents asiatiques, africains et latino-américains sont les moins bien dotés en termes d'intensité en R\&D. 


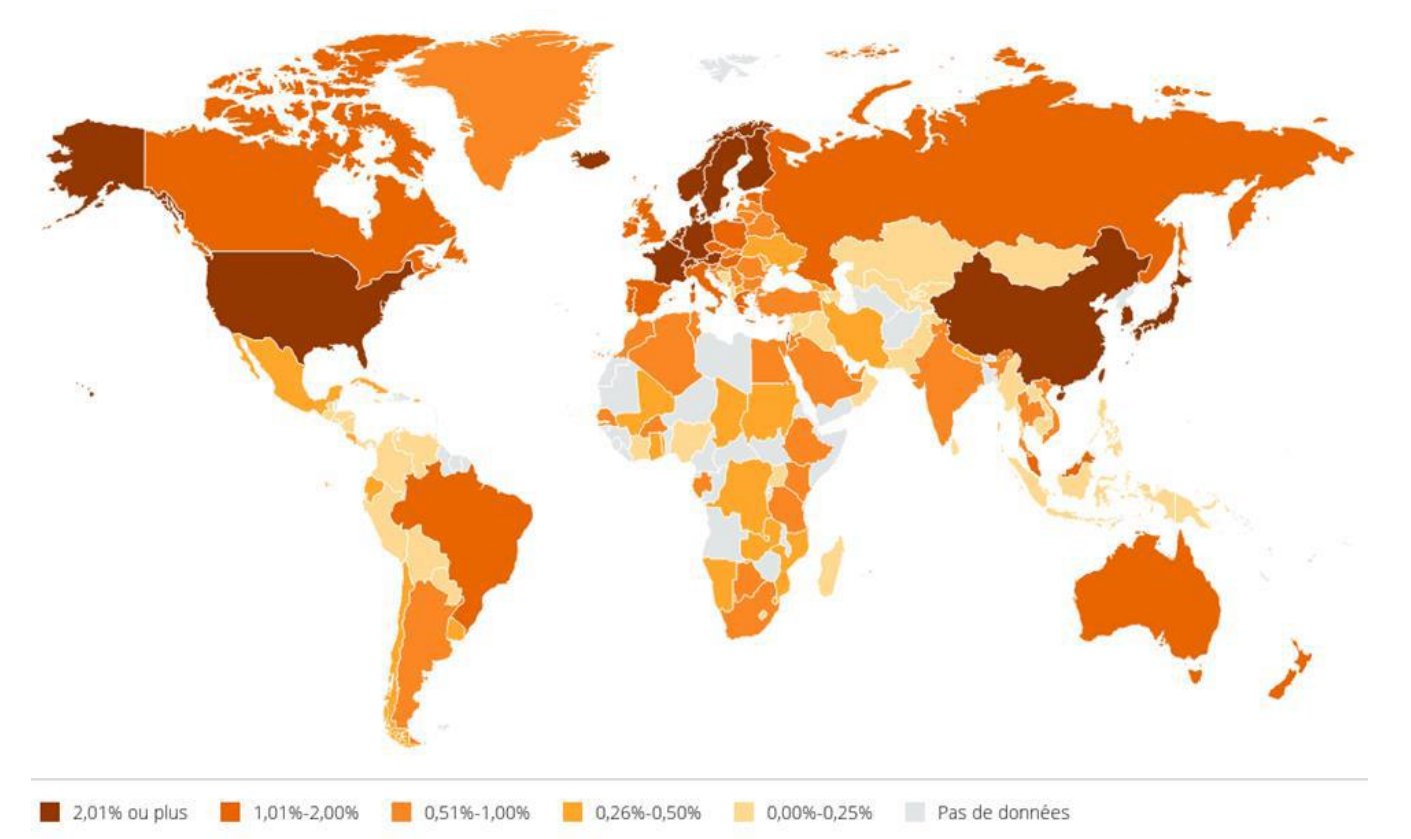

Source : UNESCO, 2019

Figure 2. Aperçu de l'intensité en $R \& D$

Si l'intensité en R\&D est insuffisante à elle-seule pour saisir la réalité très disparate des politiques scientifiques, le facteur déterminant de l'innovation qui a reçu le plus d'attention des chercheurs en économie de l'innovation ces dernières années reste la R\&D. Quant aux brevets, ils représentent un output dont les résultats ne sont guère satisfaisants dans les continents précités : l'Afrique représente $0,1 \%$ de la part des brevets de l'USPTO en 2013, l'Amérique Latine, 0,3\% et l'Asie centrale ne contribue pas du tout à cette part mondiale [UNE, 15]. Ces résultats montrent toute l'importance de la mise en place d'un transfert de technologie, dans la réalisation d'un savoir-faire et de la capacité à se réapproprier et à diffuser la technologie étrangère [CAS, 15]. Néanmoins, ces résultats doivent être rendus complémentaires avec les efforts et inputs technologiques (éducation et acquisition technologique via les I.D.E), et surtout avec des données plus sectorielles et/ou microéconomiques pour refléter de réalités locales reflétant de diverses situations d'apprentissage [CHA, 18].

\subsection{Le transfert technologique suffit-il pour améliorer le développement économique?}

Les thèses sur le transfert technologique ont été abondantes dans les années 1980. Selon Yu (1990), ce transfert est défini dans les règlements de l'O.N.U comme une diffusion de connaissances pour la fabrication d'un produit ou d'un service. D'autres définitions ont abondé durant la même période : Abot (1985) le définira plutôt comme le mouvement de science et technologie d'un groupe vers l'autre, ou Durand (1994) comme la transmission de savoirs entre des entreprises appartement à des pays différents, d'un échange d'équipements ou de techniques d'un pays à l'autre, mais essentiellement un déplacement de connaissances lié au transfert technologique. Le transfert technologique se réalise d'une entreprise à une autre d'un pays étranger, d'une nation à une autre, d'un laboratoire de recherche vers une entreprise nouvellement créée ou qui existe déjà. Si la littérature en économie de l'innovation sur le transfert technologique est riche, Hendricks (1996) résumera parfaitement les 3 catégories existantes : le transfert matériel, le transfert de concept et le transfert de connaissances :

- Le transfert de matériel est assimilé à une simple importation de nouveaux matériels et techniques associées. L'adaptation locale est réalisée sous forme d'essais/erreurs. Le cœur des technologies est emprisonné à l'intérieur de biens physiques.

- Le transfert de concept est réalisé à transfert de certains plans, formules ou livres afin de fabriquer un produit domestique. Il s'agit ici de simple transfert d'informations, données et lignes directrices pour créer des compétences de base. 
- Le transfert de connaissances scientifiques permettant la production de technologies localement adaptables. Ce transfert conduit au développement de technologies propres à partir de la technologie importée.

Teece (1976) explicitera plutôt 2 formes : une forme physique sous la caractéristique de produits et équipement divers, et une autre forme plus informationnelle qui peut être acquise si l'équipement est réellement utilisé. Le transfert de technologie a été beaucoup influencé par l'efficacité du processus d'internationalisation des firmes comme base des entreprises multinationales et l'investissement direct étranger [BUC, 76]. A une échelle macroéconomique, l'objectif d'un transfert de technologie est d'acquérir la capacité de contrôler le cœur de la technologie d'un domaine donné, afin de s'assurer une indépendance économique, de gagner en pouvoir de négociation et d'acquérir un bon niveau de compétitivité internationale [DRO, 14]. Mais à ce stade, ce transfert est statique, prend souvent la forme d'un contrat (contrat de licence de droit de propriété intellectuelle, accord de communication de savoirfaire, contrats de recherche et licence, contrat d'assistance technique et de formation professionnelle, contrat de réalisation d'ensemble industriel, contrôle d'investissement direct ou co-entreprise, etc), et n'assure que peu ou prou la formation à l'ensemble des salariés, l'amélioration des connaissances à l'ensemble d'un pays et plus largement, leur refonte institutionnelle ou infrastructurelle. Les distorsions entre acheteurs et fournisseurs sont nombreuses engendrant une inefficacité de la diffusion de technologie. Le processus d'endogénéisation de la technologie dans la production en devient limité. De plus, ce transfert est limité à un ensemble de connaissances ou matériel sur un secteur ou une entreprise donnés. Non seulement, le transfert technologique est limité par une négociation inégale ou même des critères subjectifs de choix de technologie et de partenaire, mais « l'émetteur, pour contrôler, pour réguler le processus dans le sens de ses intérêts, essayera de limiter le processus d'apprentissage du récepteur dans les sous-systèmes les "moins nobles" du système technologique " [ATA, 83]. Le transfert est alors biaisé, souvent restrictif, plutôt opportuniste, et pas toujours approprié pour les pays les moins avancés ne disposant pas d'institutions, de techniques, et du capital humain suffisants. La thèse du transfert technologique va donc, elle seule, se révéler incomplète, insuffisante et on peut se demander aujourd'hui si le transfert de technologie n'est pas devenu un argument entre les mains des pays riches pour maintenir leur domination économique. La théorie de la dépendance technologique avait été précédemment évoquée [MYT, 83], elle revient finalement progressivement dans les travaux relatifs aux obstacles desdits transferts.

\subsection{L’approche de la Science et Technologie par les Capacités Technologiques}

Il faut donc penser autrement : ne plus se restreindre au simple «transfert » mais à la capacité qu'ont les P.E.D à utiliser et à adapter les technologies existantes à des niveaux compétitifs de coûts et de qualité [LAL, 02]. Ici, l'appropriation de la technologie ne se situe plus à sa frontière mais inclut les stratégies de rattrapage mises en œuvre par les pays retardataires. La terminologie du « transfert » va ainsi progressivement céder sa place à celle de « capacité » à la fin des années 1990 [CAS, 18].

Les capacités technologiques sont essentielles afin d'utiliser la connaissance technologique et ce, pour assimiler, adapter et changer les technologies existantes [KIM, 97]. Ces dernières font référence aux efforts nationaux réalisés afin de convertir les technologies importées en leurs utilisations productives. Ce sont des ressources dynamiques, contrairement à la capacité de production qui se réfère aux équipements et machines requises pour produire des biens industriels à des niveaux d'efficience donnés [BEL, 97]. En réalité, deux aspects sont importants : en premier lieu, l'ensemble des ressources et connaissances endogènes accumulées à travers les processus d'apprentissage traditionnels [BEL, 97] et en second lieu, la capacité effective à absorber et adapter une technologie étrangère [KIM, 97]. Les processus d'apprentissage se trouvent affectés par la capacité d'absorption des firmes à combiner deux principaux éléments : la connaissance existante de base et l'intensité des efforts réalisés $[\mathrm{COH}, 89]$. La connaissance existante de base est une plate-forme essentielle de l'apprentissage technologique puisqu'elle influence directement les processus d'apprentissage et la création de 
connaissances futures. Quant à l'intensité des efforts, elle est liée à la quantité d'énergie dépensée par les acteurs organisationnels à résoudre des problèmes face aux nouvelles technologies importées.

Ces deux aspects sont complémentaires car les processus d'apprentissage au sein duquel la technologie étrangère est assimilée dépendent largement de la portée et la qualité des ressources et connaissances existantes. L'arrivée d'une technologie étrangère ne saurait par ailleurs exclure le développement des technologies endogènes existantes. Ces dernières incluent à la fois les technologies locales et importées. D'autre part, l'apprentissage technologique d'une entreprise ne se développe pas de façon isolée. Le processus d'apprentissage est riche en externalités et interrelations, notamment entre producteurs et utilisateurs [LUN, 88]. Peu de ces liens transitent par le marché et ont plutôt tendance à prendre place informellement à travers l'apprentissage routinier. Aussi, les capacités nationales d'absorption [NAR, 04] ou capacités nationales technologiques [LAL, 00] dépassent la simple somme des capacités des entreprises. De façon réciproque, la capacité à absorber les technologies n'est pas seulement fonction des firmes au sein de l'économie. Pendant que l'apprentissage et l'absorption technologique prennent place au niveau de la firme et que les autres acteurs d'un système technologique ont pour objectif central de promouvoir la capacité d'innover, le succès ou l'échec de ces dernières est orchestré de par le système entier [KIM, 97].

La focalisation sur les capacités technologiques déplace par ailleurs l'idée d'appropriation technologique à celle d'apprentissage passif et actif. Contrairement à l'idée selon laquelle les pays en développement peuvent simplement importer les technologies des pays développés et les utiliser dans la production sans réaliser d'efforts spécifiques, la notion de capacité technologique souligne que les entreprises n'ont pas la meilleure connaissance du cadre technologique disponible et la manière de créer de nouvelles technologies de manière efficiente. Trouver la technologie appropriée à des prix adéquats nécessite coûts et risques notamment au sein des pays en développement.

\subsection{L'approche de la Science et Technologie par les Capacités d'Innovation, Socio- économiques et la construction de Compétences}

Les années 2000 vont marquer les travaux en l'espèce par le basculement de " capacité technologique » vers celui de "capacité d'innovation, d'apprentissage et de compétences ». A ce titre, la dimension interactive de l'apprentissage va être essentielle. Selon Oyelaran-Oyeyinka (1997), une dynamique positive se reproduit lorsque sont établis : a) une variété de liens nécessitant offreurs, producteurs et fournisseurs de services ; b) une propension à la coopération et à la collaboration ; c) un large nombre d'acteurs diffusant l'information et la connaissance nécessaire à la production de biens et services ; d) une propension à l'incitation d'initiatives privées par la demande publique ; e) un fort réseautage local-global formel et/ou informel. Ces interactions sont capitales dans la mesure où différents types de connaissances vont pouvoir se combiner à travers de nouvelles trajectoires, et de nouvelles connaissances vont se créer. Dans la problématique de pays en développement ou de certaines économies en transition, les interactions, vectrices d'apprentissage, sont souvent inexistantes, peu nombreuses ou inappropriées. L'asymétrie d'informations conduit à de faibles flux d'informations et de connaissances parmi les acteurs du système d'innovation et les universités et laboratoires de R\&D restent très éloignés des systèmes de production. Les sphères académiques et productives demeurent faiblement intégrées les unes aux autres. Le manque d'intégration entre les centres de recherche et le système productif s'explique par deux principaux facteurs : l'accroissement exponentiel des effectifs dans les universités produit un effet d'éviction des activités de recherche au profit des activités d'enseignement. Seconde cause évoquée : l'insuffisance de grands groupes industriels conduit à l'isolement des unités de production par rapport aux centres de recherche et aux fournisseurs les privant d'effets de rétroaction sur les processus d'apprentissage. Les capacités d'innovation sont au final entravées par les difficultés des acteurs liés à la science et technologie à s'interconnecter [MUC, 03]. Ces contraintes et difficultés n'empêchent pas de donner aux capacités d'innovation une dimension spécifique dans les économies moins développées [CAS, 15]. 
Sur le plan conceptuel, les capacités d'innovation englobent celles de capacités d'apprentissage. Elles sont souvent employées dans le même sens : diffuser le savoir par des mécanismes dynamiques d'apprentissage. Ces capacités comprennent la réalisation de processus d'apprentissage par la pratique (learning by doing), par l'usage (learning by using) ou par interaction (learning by interacting) (le «DUI» mode). Mais ce ne sont pas les seuls. Edquist (2001) oppose par exemple les processus d'apprentissage organisationnels des processus d'apprentissage individuels, les deux étant indispensables à la compréhension des phénomènes d'innovation. Les processus d'apprentissage organisationnels sont des mécanismes collectifs contrôlés par les firmes et liés à la R\&D ainsi qu'aux apprentissages D.U.I. Les processus d'apprentissage individuels (éducation, formation) sont directement contrôlés par les individus et concernent le capital humain. Ce sont des prérequis inhérents aux processus d'innovation, même s'ils ne les concernent pas directement. Dans cette même perspective, Gregersen et Johnson (1997) identifient les processus d'apprentissage directs des processus d'apprentissages indirects. Les processus d'apprentissage directs visent principalement les universités, centres de recherches et laboratoires en $R \& D$ et concernent les organisations formelles. Les processus d'apprentissage indirects touchent les processus d'apprentissage routiniers : apprentissage par la pratique, usage et interaction, etc. Le point semblable entre les deux processus réside dans leur caractère social et interactif. Plus précisément, Lindegaard (1997) scinde les processus d'apprentissage directs des processus indirects au sein des institutions formelles et informelles. Ces processus d'apprentissage combinent plus globalement quatre types de connaissances [LUN, 94]: le know-what ou la connaissance informationnelle, le know-why ou la compréhension des phénomènes sociaux et naturels (connaissance scientifique), le know-who ou la capacité sociale à coopérer et à communiquer et enfin, le know-how ou la capacité à faire quelque chose à un niveau pratique (l'expérience).

Tous ces processus, loin d'être exhaustifs, sont liés à la quantité d'énergie dépensée par les acteurs à résoudre des problèmes, mais également à la connaissance qu'ont les acteurs à maitriser les nouveaux savoirs. Là aussi, il s'agit d'efforts et de trajectoires d'utilisation de la connaissance locale par la capacité à (pouvoir) internaliser les connaissances étrangères. On parle de domestication des connaissances, de réadaptation et de réappropriation locale. Pour cela, si l'utilisation des connaissances se réalise par la construction de capacités, elle dépend d'opportunités d'apprentissage. Le développement d'une entreprise, région ou nation ne saurait évoluer autrement [NIE, 05]. Autrement dit, la dynamique d'apprentissage se construit par l'existence d'opportunités et de capacités d'apprentissage [CAS, 15]. Comme mentionné, les capacités d'apprentissage représentent la connaissance existante, requise, l'expérience ou l'organisation de pratiques routinières. Ces capacités, basées sur une demande en constante évolution, se développent très rapidement au sein de contextes d'opportunités d'apprentissage : c'est-à-dire l'accès aux universités, aux centres et institutions de recherche, mais aussi aux systèmes éducatifs ou projets de recherche nationaux. Si les capacités permettent la diffusion et la création de connaissances, les opportunités d'apprentissage sont relatives à l'application et l'utilisation de ces dernières [ARO, 03]. Le premier élément est relatif à la construction de capacités à travers la recherche de solutions nouvelles (ou améliorées) et le second à l'utilisation de ces capacités.

Enfin, dernière terminologie développée récemment dans les travaux de la technologie inclusive : les capacités socio-économiques. Sa genèse remonte aux travaux de Lundvall et al., (2002) qui ont associé l'innovation aux capacités socio-économiques telles que définies par Amartya Sen. Les capacités socio-économiques (les capabilities) relient la croissance économique aux processus d'expansion des capacités humaines de vivre des vies plus riches et plus libres. L'apprentissage n'est plus uniquement une valeur instrumentale mais bien substantive à la qualité de vie des individus. Cette valeur va de pair avec la rapidité des nouvelles compétences et capacités à acquérir pour s'approprier les connaissances technologiques. On s'intéresse plus concrètement aux conditions de vie et à leurs liens sur l'apprentissage et la technologie [JOH, 03]. En parallèle, et dans cette large approche d'appropriation des connaissances, la terminologie de « construction de compétences » [MUC, 03] va 
revêtir une importance particulière car son intérêt est d'attacher plus d'attention à l'utilisation des connaissances (et leur condition) qu'à leur diffusion [CHA, 18]. Le tableau ci-dessous résume les principales terminologies quant au sens donné à l'appropriation des connaissances des pays en développement ou en transition. Nous retenons donc, à la lumière des derniers travaux (Tableau 1), que la science et technologie dépend de la prédisposition de ces pays à établir, au-delà du strict transfert de connaissances, de larges capacités socio-économiques.

\begin{tabular}{|c|c|c|c|}
\hline Terminologie & Caractérisation & Sens & Méthodologie \\
\hline $\begin{array}{l}\text { Transfert de technologie } \\
\text { [TEE., 76] }\end{array}$ & $\begin{array}{lr}\text { Mécanisme } & \text { de } \\
\text { réappropriation de } & \text { la } \\
\text { technologie étrangère } & \end{array}$ & $\begin{array}{l}\text { Etroit : Apprentissage } \\
\text { technologique }\end{array}$ & Micro/Méso \\
\hline $\begin{array}{l}\text { Capacité technologique } \\
{\left[\begin{array}{l}\text { LAL, 92, KIM, 97, } \\
\text { OGB, 95] }\end{array}\right.}\end{array}$ & $\begin{array}{l}\text { Efforts réalisés pour } \\
\text { diffuser et utiliser les } \\
\text { technologies étrangères }\end{array}$ & $\begin{array}{l}\text { Etroit : Apprentissage } \\
\text { technologique }\end{array}$ & Micro/Méso/Macro \\
\hline $\begin{array}{l}\text { Capacité d'apprentissage } \\
{[\text { LUN, 02] }}\end{array}$ & $\begin{array}{l}\text { Efforts pour instituer une } \\
\text { Learning Economy }\end{array}$ & $\begin{array}{l}\text { Large: Apprentissage } \\
\text { technologique, } \\
\text { institutionnel, sociétal }\end{array}$ & Micro/Méso/Macro \\
\hline $\begin{array}{l}\text { Capacité d'innovation } \\
{[\mathrm{JOH}, 03]}\end{array}$ & $\begin{array}{lr}\text { Efforts pour créer, } \\
\text { diffuser, utiliser } \\
\text { connaissances }\end{array}$ & $\begin{array}{l}\text { Large: Apprentissage } \\
\text { technologique, } \\
\text { institutionnel, sociétal }\end{array}$ & Micro/Méso/Macro \\
\hline $\begin{array}{l}\text { Construction de } \\
\text { compétences [MUC, 03] }\end{array}$ & $\begin{array}{lr}\text { Efforts pour créer, } \\
\text { diffuser, utiliser les } \\
\text { connaissances : focus } & \text { sur } \\
\text { l'utilisation } & \text { des } \\
\text { connaissances } & \end{array}$ & $\begin{array}{ll}\text { Large : focus sur } \\
\text { l'utilisation de la } \\
\text { connaissance }\end{array}$ & Micro/Méso/Macro \\
\hline $\begin{array}{l}\text { Capacités socio- } \\
\text { économiques [CHA, 18] }\end{array}$ & $\begin{array}{l}\text { Efforts sociétaux pour } \\
\text { améliorer le bien-être des } \\
\text { populations }\end{array}$ & Large (sociétal) & Macro \\
\hline
\end{tabular}

Tableau 1. Du transfert technologique à la construction de capacités

\section{La Science et Technologie dans les théories du Développement Economique}

\subsection{La légitimité du couple science/développement}

Deux aspects sont à considérer avant de pouvoir s'intéresser à la relation entre la S\&T et le développement économique : il s'agit de l'introduction de la S\&T dans les théories du développement économique et du développement économique comme facteur de promotion de la S\&T.

S'il reste incontestable que les théories de la croissance endogène ont permis d'introduire le progrès technologique et la formation du capital humain dans les modèles de croissance et ont consolidé le rôle de la technologie dans la croissance économique, elles demeurent limitées dans la conception de la science, dont l'importance a été marquée par les travaux évolutionnistes de l'innovation systémique dans les années 1980. On évoque alors le terme de «capacité manquante » (« missing capability») pour justifier la nécessité de créer une nouvelle voie d'entrée l'apprentissage dans les stratégies de développement [JOH, 03]. En ce sens, la capacité technologique devient une composante essentielle du développement économique [JUM, 01]. Dans une perspective inverse, si la S\&T ne se sont pas introduites dans les théories du développement, l'approche systémique de l'innovation a, quant à elle, été particulièrement influencée par les thèses développementalistes. Les théories du développement 
deviennent de nouveaux champs d'application des capacités d'innovation [LUN, 02]. Tandis que l'innovation systémique puise chez Hirschman (1958) et Steward (1977) la notion de rétroaction systémique [KLI, 86], elle emprunte les idées de rétroactions positives ou négatives, de « causalités circulaires cumulatives » et de cercles vicieux ou vertueux à Myrdal (1968). C'est également chez Hirshman (1981), dans son illustre essai « Essays in trespassing. Economics to politics and beyond », qu'on retrouve le caractère interdisciplinaire de l'approche [EDQ, 97]. L'intérêt porté aux processus interactifs de l'innovation [LUN, 88] provient également des écrits d'Hirschman (1981) : " le développement est essentiellement le récit de la manière dont les choses mènent à de nouvelles choses, et d'un point de vue spécifique, les liens représentent ce récit» [HIR, 81, p.75]. La notion de capacité réside précisément dans le fait que la connaissance et le contexte politique et socio-économique doivent être pris en compte tout en n'écartant pas le pouvoir des relations entre ces derniers dans l'accumulation des connaissances [CAS, 14]. Si la connaissance et le contexte socio-économique sont historiquement déterminés, la relation entre le développement économique et le changement technique est établie par la croissance, basée sur l'accumulation de connaissances et la compréhension du développement économique par une vision systémique [FUR, 61]. En définitive, les idées conductrices de l'économie du développement se trouvent réexportées dans les travaux sur les capacités d'innovation.

Le processus de développement est caractérisé par des changements majeurs réalisés à partir de discontinuités provoquées par la structure sociale, politique et institutionnelle de chaque Etat. Le développement est donc un processus systémique, créant un développement inégal dans le monde. Pour Furtado (1961), c'est à partir de ces changements structurels économiques et sociaux que l'essence du développement réside. Furtado a établi une relation directe entre le développement économique et le progrès technologique en accentuant le fait que la croissance et l'économie sont basées sur l'accumulation de la connaissance scientifique et la capacité par la connaissance à résoudre des problèmes pratiques. Comme le comportement des variables économiques est relié à des paramètres qui sont définis sur un contexte historique spécifique, il est impossible d'isoler l'étude du phénomène économique de sa base historique. Le sous-développement n'est donc pas une anomalie mais un modèle de fonctionnement et une évolution spécifique de l'Etat en question.

De là, l'accumulation de la connaissance, façonne le développement économique tout comme le développement économique conditionne la constitution de capacités [CAS, 15]. La réduction de la pauvreté et l'amélioration de la distribution des revenus demeurent liées à la capacité des pays pauvres à maitriser l'utilisation, la diffusion et la création des connaissances. La question des inégalités et de la pauvreté est essentielle dans les stratégies d'innovation des pays en développement en transition [COZ, 09]. Ces problèmes sont d'ailleurs ancrés dans le contexte socio-économique de ces pays. La question du développement couplée à la $\mathrm{S} \& \mathrm{~T}$ est importante à double titre. D'une part, la science et technologie renforcent les inégalités, dans la mesure où le changement technologique renforce les écarts de distribution de revenus. Le progrès technique exerce une pression considérable sur les inégalités salariales et le chômage : directement par la concurrence par les coûts que subissent les travailleurs exposés et par la suppression d'emplois dont le produit correspondant est remplacé par des importations et indirectement en stimulant l'innovation dans les pays riches afin qu'ils puissent compenser leurs couts salariaux élevés par une productivité du travail supérieure et concurrencer les pays pauvres par la qualité et la variété de leurs produits. Mais les inégalités elles-aussi, bloquent la diffusion de technologie par leur importance dans les sources de l'apprentissage (accès à l'éducation supérieure, qualité de l'enseignement, importance du système de santé, etc.). L'existence de fortes inégalités dans les relations de pouvoir, par région/pays, dans la taille des firmes, la qualité des infrastructures, les revenus par ménage, freine indéniablement les capacités d'apprentissage et d'innovation des acteurs économiques [UZU, 12]. On parle de dark side innovation [CHA, 18] pour montrer les effets néfastes de la science et technologie sur certains groupes sociaux. Si la science et la technologie améliorent les conditions économiques et sociales de certaines classes sociales, elles en excluent naturellement d'autres. La science et la technologie sont d'ailleurs souvent comprises comme 
exclusivement réservées à l'élite (capitalistes, managers, scientifiques, ingénieurs et politiciens) sans considérer les effets qu'ils génèrent sur les autres classes sociales.

\subsection{Travaux empiriques et orientation des capacités socio-économiques}

La science et la technologie sont, à travers les acteurs qu'elles représentent, des composantes actives du processus de développement économique. Les travaux empiriques vont également le prouver. Lundvall et al. (2002) ont par exemple démontré la pertinence de la combinaison apprentissage/développement au Danemark. Les problématiques d'inégalités et de lutte contre la pauvreté sont ici liées au caractère systémique de la science et technologique. Narayan et Petecsh (2002) ont également montré comment l'écoute des pays pauvres est impérative pour lier la S\&T et le développement des P.E.D. Il ne s'agit pas d'écouter la voix des pays occidentaux sur les politiques à suivre mais plutôt de faire remonter les problèmes sociétaux de la société civile. Si tel est le cas, l'émergence de systèmes technologiques informels prendrait toute leur légitimité, comme a pu le montrer Muller en Tanzanie [MUL, 11].

Dans certains pays en développement ou en transition, le traitement inégal des minorités et la mauvaise gouvernance locale à travers les problèmes de corruption détournent la construction de capital social et limitent automatiquement les capacités d'apprentissage de la société. L'économie de rente et le clientélisme souvent prégnant ne favorisent pas la création de savoirs, tout comme les stratégies gouvernementales d'élites peu formées et souvent tournées autour d'ambitions personnelles des dirigeants. Les systèmes technologiques ne représentent pas seulement des ressources pour promouvoir la recherche technologique mais bien un ensemble d'institutions qui soutiennent l'apprentissage interactif dans un objectif, tel que conceptualisée par Sen (2000), de libertés et capacités socio-économiques. Dans cette dimension, un article de Fagerberg et Shrolec (2008) montre le lien entre la science, la technologie et les capacités socio-économiques [SEN, 00]. Ces derniers se proposent d'identifier empiriquement les capacités socio-économiques à partir des éléments suivants (Tableau 2).

\begin{tabular}{|c|c|c|}
\hline Instruments & Mesures & Capacités socio-économiques \\
\hline Science, recherche, innovation & Publications scientifiques, brevets & Technologique \\
\hline Degré d'ouverture & IDE, Licences, immigration & Technologique \\
\hline Qualité de production/Normes & Normes internationales (ISO) & Technologique \\
\hline Infrastructures technologiques & $\begin{array}{l}\text { Télécommunication, internet, } \\
\text { ordinateurs }\end{array}$ & Technologique \\
\hline Capacités & $\begin{array}{l}\text { Education primaire, secondaire et } \\
\text { supérieure, compétences managériales } \\
\text { et techniques }\end{array}$ & Technologique et sociale \\
\hline Finance & $\begin{array}{l}\text { Accès au crédit bancaire, venture } \\
\text { capital }\end{array}$ & Technologique et sociale \\
\hline Qualité de gouvernance & $\begin{array}{l}\text { Corruption, lois, indépendance et } \\
\text { séparation des pouvoirs, droit de } \\
\text { propriété, régulation }\end{array}$ & Sociale \\
\hline Valeurs sociales & $\begin{array}{l}\text { Activités civiques, confiance et } \\
\text { tolérance }\end{array}$ & Sociale \\
\hline Type de système politique & Droits politiques, démocratie & Sociale \\
\hline
\end{tabular}

Source : Fabergerg et Srholec (2008) 
A partir de ces éléments, et à travers un modèle de régression proposé, les auteurs précités en concluent que le rattrapage des P.E.D se base sur 4 éléments essentiels liés au développement des systèmes technologiques, à la qualité de la gouvernance, au caractère des systèmes politiques et au degré d'ouverture des relations commerciales et investissement directs étrangers. Cela montre l'importance des capacités socio-économiques dans la construction d'un développement pérenne. Encourager les acteurs à avoir une attitude positive alors que ces derniers n'ont pour la plupart pas confiance aux institutions permet de contribuer à un développement tel que conceptualisé par Amartya Sen (2000) comme fin et moyen.

Dans un autre axe, se pose la question du rôle de la S\&T dans le développement durable. La compréhension du rôle de la technologie dans et pour le développement durable n'est pas nouvelle et a été analysée par Freeman dans les années 1970 [PER, 15]. Dans son Economics of Hope (1992), il avait préalablement rappelé l'intérêt d'un paradigme techno-économique vert. A partir de technologies durables, il a donc fallu trouver des solutions technologiques, sociales ou institutionnelles pour réduire l'impact environnemental sur l'activité humaine [CHA, 18]. Dans les systèmes d'innovation et technologiques, le S.I durable est constitué par des éléments sociaux, humains et naturels ainsi que par des relations qui interagissent dans la production, diffusion et utilisation de la connaissance nouvelle et économiquement profitable [SEG, 03]. On va ainsi retrouver les 4 formes de ressources tangibles/intangibles comme fondement de nouvelles méthodologies proposées (Tableau 3). Toutefois, la littérature sur les systèmes technologiques durables a tendance à focaliser son intérêt sur une seule technologie (biomasse, solaire, véhicule électrique, etc.) ou sur un cluster technologique, sans tenir compte des relations complexes entre ces technologies et les services écosystémiques [CHA, 18]. Plus globalement, l'intérêt est désormais de comprendre les relations complexes entre les systèmes technologiques et les systèmes socio-écologiques sur une échelle macro-économique.

\begin{tabular}{|l|l|l|}
\hline & $\begin{array}{l}\text { Ressources facilement } \\
\text { reproductibles }\end{array}$ & $\begin{array}{l}\text { Ressources difficilement } \\
\text { reproductibles }\end{array}$ \\
\hline Ressources tangibles & Capital productif [LUN, 02] & Capital naturel [SEG, 03] \\
\hline Ressources intangibles & Capital intellectuel [LAM, 07] & Capital social [HEE, 14] \\
\hline
\end{tabular}

Source : adapté de Lundvall et al., (2002)

Tableau 3. Les différentes ressources pour la diffusion des technologies

Conclusion : Quelle politique technologique à des fins de développement économique ?

Dans la problématique des pays en développement et en transition, la reconnaissance du rôle de la S\&T dans le développement économique et social est importante à double titre :

Tout d'abord, les entreprises recherchant constamment des liens plus étroits avec la base scientifique, et le progrès technique s'étant accéléré dans les domaines où l'innovation repose sur la science (biotechnologies, technologies de l'information et nouveaux matériaux), la création de capacités (institutionnelles, socio-économiques, technologiques) permet de démocratiser les technologies, de réduire notablement les coûts et dans une autre mesure, d'accéder à un meilleur confort de vie et bien être. Mais des inégalités peuvent également se creuser dans la mesure où « des changements sur la capacité à utiliser des données informatisées pourraient notamment accroître la polarisation du marché du travail entre la main-d'œuvre hautement qualifiée et la main-d'œuvre peu qualifiée » [ONU, 17]. Cette dark side innovation est importante à relater, surtout dans les pays où l'appropriation des technologies est fragile et limitée à une diaspora scientifique. 
Dans un second temps, le développement économique va pleinement jouer sur le potentiel de science et technologie à travers le niveau d'instruction globale, la qualité des infrastructures et l'ensemble du cadre institutionnel de la $S \& T$ à travers la $R \& D$ et autres institutions apprenantes (Encadré 2). Alors, est-ce le développement économique qui promeut en premier lieu le potentiel de S\&T ou au contraire, la $S \& T$ qui influence le niveau de développement économique ? La réponse est évidemment peu généralisable, selon le pays visité, le niveau de développement, le contexte politique et historique et les politiques technologiques et scientifiques mises en place [CAS, 17a].

Au final, la substitution du transfert stricto sensu vers un besoin d'un développement S\&T lié aux capacités et compétences locales sont parfaitement cohérents avec l'axe 9 des ODD reposant sur l'innovation et le progrès technique. L'industrialisation ou l'agriculture durable nécessitent la construction de capacités, lesquelles seront réalisables par le biais de rapprochements entre capacités académiques et entrepreneurs du Sud. Et ces liens, créateurs de systèmes d'innovation, rentrent parfaitement dans l'axe 17 des ODD, sur la nécessité de coopérations et partenariats internationaux.

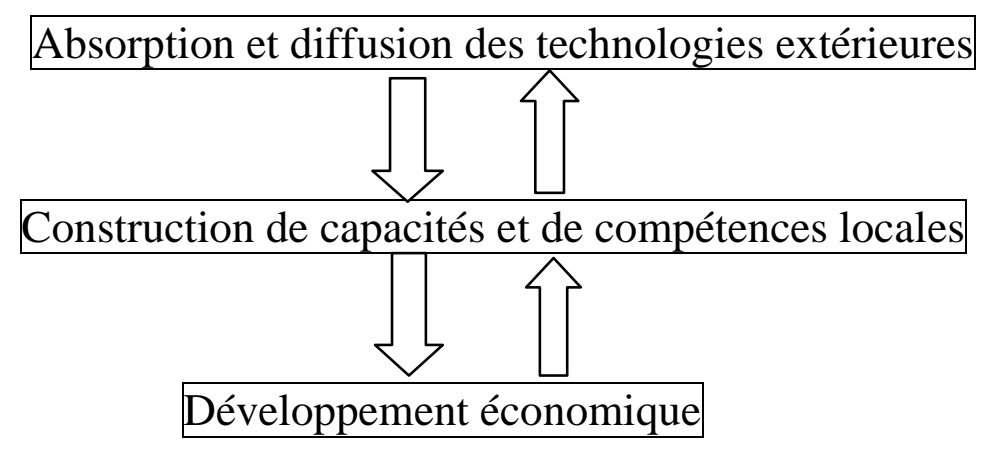

Encadré 2. Les orientations des politiques de S\&T dans un objectif de développement économique

Ces nouveaux enjeux interrogent également sur la définition d'une politique technologique : quelle politique technologique semble être la plus appropriée en termes de développement et de progrès social ? Traditionnellement, l'Etat met en œuvre des mesures permettant la coopération et la synergie entre tous les acteurs pour générer des ressources technologiques. Ces mesures conduisent également à l'amélioration de l'attractivité économique et technologique nationale. En toute évidence, l'intervention publique en matière de politique d'innovation ne se limite pas seulement en termes de brevet. Elle cherche à favoriser les coopérations et les échanges de connaissances entre différents acteurs liés à la science et la technologique [CAS, 17b]. L'élaboration d'une politique de S\&T porte sur la prise en compte de la nature évolutive, dynamique interactive des processus d'innovation [LEI, 10]. Cette nature, caractérisée par la diversité des interactions, des collaborations et de leur intensité est en premier lieu un résultat issu des conditions de formulation des politiques, mais surtout de leur mise en œuvre et de leur évaluation. En second lieu, elle résulte des institutions de coordination sociales spécifiques aux différentes cultures et sociétés. Au final, les politiques de S\&T doivent faciliter les interactions entre utilisateurs de technologies, et ce, en créant différentes situations d'apprentissage qui massifient les mécanismes d'imitation et de dissémination des technologies tout en constituant un solide socle de capacités socioéconomiques.

\section{Bibliographie}

[ABO, 85] ABOT P.G., "Technology Transfer in the Construction Industry, Infrastructure and Industrial development", The Economist Intelligence, Special Report, No 223, 1985. 
[ARO, 03] AROCENA R., SUTZ J., Understanding underdevelopment today: news perspectives on NSI, GLOBAL Network for Economic of Learning, Innovation and Competence Building Systems, Brésil, 2003.

[ATA, 83] ATAMER T., «Le processus d'acquisition technologique par les entreprises d'un pays semi-industrialisé Deux études de cas », Revue d'économie industrielle, vol. 26, 4e trimestre 1983. p. 24-33, 1983.

[BEL, 97] BELL M., PAVITT K., “Technological accumulation and industrial growth: contrast between developed and developing countries", dans ARCHIBUGI D., MICHIE J., Technology, Globalization and Economic Performance, Cambridge University Press, p. 83-138, 1997.

[BUC, 76] BUCHLEY P., CASSON M., The future of multinational enterprise, MacMillan, Londres, 1976.

[CAS, 15] CASADELlA V., LIU Z., UZUNIDIS D., Innovation Capabilities and economic development in open economics, Iste Editions, Vol 1., Wiley, 2015.

[CAS, 17a] CASADELLA V., TAHI S., «Capacités et politiques d'innovation dans les pays moins avancés : enseignements tirés du cas du Sénégal », Innovations, vol. 53, no. 2, 2017, p. 13-39, 2017.

[CAS, 17b] CASADELLA V., UZUNIDIS D., "National Innovation Systems of the South, Innovation and Economic Development Policies: A Multidimensional Approach", Journal of Innovation Economics \& Management, vol. 23, no. 2, 2017, p. 137-157, 2017.

[CAS, 18] CASADELLA V., UZUNIDIS D., Innovation Capabilities as a Prerequisite for Forming a National Innovation System, Tech Inn, 2018/03, DOI : 10.21494/ISTE.OP.2018.0239, 2018.

[CAS, 14] CASSIOLATO J.E, MATOS M.P., LASTRES H.M., Innovation systems and development, dans CURRIEALDER B., KANBUR R., MALONE D.M., International development: ideas, experience and prospects, Oxford University Press, 2014.

[CHA, 18] CHAMINADE C., LUNDVALL B.A., HANEEF S., Advanced Introduction to National Innovation Systems, Elgar Publishing, 2018.

[COH, 89] COHEN W., LEVINTHAL D., Innovation and learning: the two faces of R\&D, Economic Journal, 99, p. 569596, 1989.

[COZ, 09] COZZENS S.E., KAPLINSKY R., Innovation, poverty and inequality: cause, coincidence of co evolution? Dans LUNDVALL B.A., JOSEPH K.J., CHAMINADE C., VANG J., Handbook of Innovation systems and developing countries, Elgar Publishing, Cheltelham, p. 57-83, 2009.

[DRO, 14] DROUVOT H., VERNAT G., Les politiques de développement technologique, Editions de l'IHEAL, 2014.

[DUR, 94] DURAND C., La coopération technologique internationale, De Boech éditions, 1994.

[EDQ,97] EDQUIST C., Systems of Innovation, Technologies, Institutions and Organizations. Pinter. Londres, 1997.

[EDQ, 01] Edquist C., System of Innovation for Development, UNIDO World Industrial Development Report (WIRD), 2001.

[FAG,08] FAGERBERG J., SHROLEC M., National Innovation systems, capabilities and economic development, Research Policy, 37: p. 1417-1435, 2008.

[FAO, 20] FAO, Suivi des progrès accomplis dans le cadre des indicateurs d'ODD lié à l'alimentation et l'agriculture en 2020. Document disponible sur http://www.fao.org/sdg-progress-report/fr/. 2020

[FUR, 61] FURTADO C., Desenvolvimento e subdesenvolvimento, Rio de Janeiro, ed. Fundo de Cultura, 1961.

[GRE, 97] GREGERSEN B., JOHNSON B., Learning Economies, Innovation Systems and European Integration, Regional Studies, Vol 31 no 5, 1997.

[HEE, 14] HEEKS R., FOSTER C., NUGROHO Y., New models of inclusive innovation for development, Innov. Dev. 4 (2), p. 175-185, 2014.

[HEN, 96] HENDRICKS C., Problématique du transfert de technologie et nouvelles theories de l'innovation et de la firme, Revue Région et Développement, n3, 1996.

[HIR, 58] HIRSCHMAN A.O., The strategy of economic development, Yale University Press, New Haven. UK, 1958.

[HIR, 81] HIRSCHMAN A.O., Essays in trespassing. Economics to Politics and beyond. Cambridge University Press, Cambridge, 1981.

[JOH, 03] JOHNSON B., LUNDVALL B.A., National System of Innovation and Economic development, dans MUCHIE M., GAMMELTOFT P., LUNDVALL B.A., Putting Africa First: the making of African Innovation Systems, Aalborg University Press. Danemark, p.13-29, 2003.

(c) 2021 ISTE OpenScience - Published by ISTE Ltd. London, UK - openscience.fr 
[JUM, 01] JUMA C.F., HONCA D., HUETE-PEREZ J., KONDE V., LEE S., Global governance of technology: meeting the needs of developing countries, International journal of technology management, Vol. 22, $\mathrm{N}^{\circ} 7-8,2001$.

[KIM, 97] KIM L., Imitation to innovation. The dynamics of Korea's technological learning, Harvard university press, Boston MA. USA, 1997.

[KLI, 86] KLINE S.J., ROSENBERG N., An overview of innovation, dans ROSENBERG N., LANDAU R. (Eds.), The positive sum strategy: harnessing technology for economic growth, National Academy Press, Washington, p.275-305, 1986.

[LAL, 00] LALL S., Technological Change and Industrialization in the Asian Newly Industrializing Economies: Achievements and Challenges, dans KIM L., NELSON R. Technology, Learning and Innovation, Cambridge University Press, p.13-69, 2000.

[LAL, 02] Lall S., Pietrobelli C., (2002), Falling to compete. Technology development and technology systems in Africa. Elgar. Cheltenham.

[LAM, 07] LAM A., LUNDVALL B.A., 'The Learning organisation and national systems of competence building and innovation', in N. Lorenz and B-A Lundvall (eds) How Europe's Economies Learn: Coordinating Competing Models, Oxford University Press, p. 110-139, 2007.

[LIN, 97] LINDEGAARD K., State of the art of innovation system analysis, DRUID Working Paper. Aalborg School. Danemark, 1997.

[LUN, 88] LUNDVALL B.A., Innovation as an interactive process: form user-producer interaction to the national system of innovation dans DOSI G., FREEMAN C., NELSON R., SILVERBERG G., SOETE L., Technical change and Economic Theory. Pinter. Londres, p.349-369, 1988.

[LUN, 94] LUNDVALL B.A., JOHNSON B., The learning Economy, Journal of industry Studies, Vol1, Number2, 2343, 1994.

[LUN, 02] LUNDVALL B.A., JOHNSON B., ANDERSEN E.S., DALUM B., National systems of production, innovation and competence building, Research Policy, Vol.31, Issue 2, February 2002, p. 213-231, 2002.

[LOG, 17] LOGAN C., Synthèse de politique $n^{\circ} 39$, l'ambition des ODD confronté à la réalité : la justice demeure inaccessible à beaucoup d'africains, Afrobaromètre, 2017.

[NAR, 02] NARAYAN N., PETESCH Voices of the poor: from many lands, New York. Oxford University Press, 2002.

[NAR, 04] NARULA R., Understanding absorptive capacities in a Innovation Systems context: Consequences for Economic and Employment Growth, DRUID Working Paper Nº4-02. Aalborg School. Danemark, 2004.

[MYT, 83] MYTELKA L.K., Le capitalisme fondé sur la connaissance et le changement dans les stratégies des entreprises industrielles. Études Internationales, 14 (3), p. 433-452. https://doi.org/10.7202/701538ar. 1983.

[MUC, 03] MUCHIE M., GAMMELTOFT P., LUNDVALL B.A., The making of African Innovation Systems, Aalbord University Press, 2003.

[MUL, 01] MULLER J., Making Ends Meet: Local socio-technological transformations in the South: based on case studies from Tanzania. Aalborg: Department of Development and Planning, Aalborg University, 2011.

[NIE, 05] NIELSEN B.B., The role of knowledge embeddedness in the creation of synergies in strategic alliances, Journal of Business Research, 58, p.1194-204, 2005.

[OGB, 95] OGBU O.M., OYELARAN-OYEYINKA B., MLAWA H.,Understanding desindustrialization and technological stagnation in Sub-Saharan Africa: A framework, In Technology Policy and Practice in Africa: IDRC. Canada, 1995.

[ONU, 17] ONU, Note du secretariat de la CNUCED, La science, la technologie et l'innovation en tant que moyens de favoriser la réalisation des objectifs de développement durable, 2017.

[OYE, 97] Oyelaran-Oyeyinka B., Nnewi: an emergent industrial cluster in Nigeria. Ibadan: Technopol, 1997.

[PER, 15] PEREZ C., The new context for industrializing around natural resources: an opportunity for Latin America (and other resource rich countries), Technology Governance and Economic Dynamics, Working Paper, Vol.62, 2015.

[SEG, 03] SEGURA-BONILLA O., Competitiveness, systems of innovation and the learning economy: the forest sector in Costa Rica, Forest Policy and Economics 5, 73-384, 2003.

[SEN, 00] SEN A., (2000), Development as Freedom, Anchor Books, New York.

[STE, 77] STEWARD F., Technology and underdevelopment, Macmillan, Londres, 1977. 
[TEE, 76] TEECE D., The Multinational Corporation and the Resource Cost of International Technology Transfer, Cambridge, MA: Ballinger, 1976.

[UNE, 15] UNESCO, Rapport de l'Unesco sur la science, vers 2030. Editions Unesco. Paris, 2015.

[UNE, 19] UNESCO, Investissements mondiaux en R\&D, Fiche d'information $n^{\circ} 54,2019$.

[UZU, 12] UZUNIDIS D., BOUTILLIER S., Globalization of R\&D and network innovation: what do we learn from the evolutionist theory? Innovations, Journal of Innovation Economics and Management, 10(2), p. 23-52, 2012.

[YU, 90] Yu X.Y., International Economic Laws, Nanjing, International Press, 1990. 